Observational Report

\title{
National Perioperative Outcomes for Intrathecal Pump, Spinal Cord Stimulator, and Peripheral Nerve Stimulator Procedures
}

Christopher R. Abrecht, MD¹, Rodney A. Gabriel, MD¹, Richard P. Dutton, MD², Alan D. Kaye, MD, PhD³ , Edward Michna, MD, JD', and Richard D. Urman, MD ${ }^{1}$

From: ${ }^{1}$ Brigham and Women's Hospital, Department of Anesthesiology, Perioperative, and Pain Medicine, Boston, MA; ${ }^{2}$ Anesthesia Quality Institute, Executive Director, American Society of Anesthesiologists, Chief Quality Officer; ${ }^{3}$ Louisiana

State University Health Sciences Center, Department of Anesthesiology Department of Pharmacology

Address Correspondence: Richard D. Urman

Brigham and Women's Hospital

Department of

Anesthesiology, Perioperative, and Pain Medicine 75 Francis Streeet Boston, MA

rurman@pa

Disclaimer: There was no external funding in the preparation of this manuscript.

Conflict of interest: Each author certifies that he or she, or a member of his or her immediate family, has no commercial association

(i.e., consultancies,

stock ownership, equity interest, patent/licensing arrangements, etc.) that might pose a conflict of interest in connection with the submitted manuscript.

Manuscript received: 05-22-2015 Accepted for publication: 05-29-2015

Free full manuscript: www.painphysicianjournal.

com
Background: There is abundant literature on the long-term complications of intrathecal pumps (ITP), spinal cord stimulators (SCS), and peripheral nerve stimulators (PNS) used in the treatment of chronic pain. There is less information, however, on the perioperative complications of these procedures.

Objective: Exploration of the perioperative outcomes of implantable pain devices

Study Design: Observational study.

Setting: University hospitals, community hospitals, specialty hospitals, attached surgery centers, and freestanding surgery centers

Methods: Data were obtained from the National Anesthesia Clinical Outcomes Registry (NACOR) of the Anesthesia Quality Institute (AQI). Information was collected on patient demographics, procedure information, anesthetic administered, diagnosis linked to the procedure, and perioperative outcomes.

Results: The search yielded 12,611 ITP, 19,276 SCS, and 15,184 PNS cases from 2010 to 2014. In this sample, the majority of procedures were performed at community hospitals, not university medical centers. The most common diagnosis cited for an ITP was an implant complication $(n=2,570)$, followed by spasticity, and non-malignant back pain. For SCS, the most common diagnoses were lower back pain $(n=5,515)$ or radiculopathy $(n=2,398)$. For PNS, by far the most common diagnosis related to urinary dysfunction $(n=8,745)$, with painful bladder syndrome a small minority $(n=133)$. General anesthetics were more often performed for ITP than for SCS and PNS procedures (60.6\% vs. $31.8 \%$ and $32.2 \%$, respectively). Hemodynamic instability was a common outcome (13.9\% for ITP procedures); other common outcomes for all the procedures included case delays, inadequate pain control, and extended PACU stays.

Limitations: Despite the large sample size in this study, not all medical centers transmit their outcome data to NACOR. Furthermore, some institutions do not report clinical outcomes for every case to NACOR, making the sample size of assessing complications smaller and potentially more biased. Finally, procedures identified in the NACOR database using CPT may be similar but not identical and therefore potentially influence outcomes.

Conclusions: Databases such as NACOR can provide rich information on ITP, SCS, and PNS for physicians performing these procedures. In this sample, ITP procedures, performed on the patients with the most severe cormobidities and often-requiring general anesthesia, were the most likely to be associated with hemodynamic instability, inadequate pain control, and extended PACU stays. Complications relating to the ITP are also the most common reason for an operation. These findings underscore the importance of proper patient selection for ITP and other implantable pain devices, in particular for patients with malignant pain or multiple co-morbidities. To identify the root causes of complications, additional information is needed on the procedure performed (e.g., an implant vs a revision), the surgical technique used, and the device implanted, as well as on specific patient comorbidities. Such information will likely become more available as resources like NACOR expand and as electronic medical record systems and coding become more integrated.

Key words: Perioperative outcomes of implantable pain devices, National Anesthesia Clinical Outcomes Registry, Anesthesia Quality Institute, intrathecal pumps, spinal cord stimulators, peripheral nerve stimulators

Pain Physician 2015; 18:547-554 
nvasive procedures to treat chronic pain have been increasing at a significant rate in recent years. An analysis of Medicare beneficiaries found, for instance, there has been a $197 \%$ increase in interventional pain procedures from 1997 to 2006 (1). The increasing prominence of chronic pain management has also been reflected in closed claims analysis. The American Society of Anesthesiologists Closed Claims Project has reported, for example, that chronic pain represented $5 \%$ of all claims in the 1980 s but $18 \%$ of all claims from 2000 to 2007 (2). While some invasive pain procedures such as cervical blocks and injections have been studied in detail (3), to date there has been no such robust claims analysis for intrathecal pumps (ITP), implantable spinal cord stimulators (SCS), or peripheral nerve stimulators (PNS) in the perioperative setting.

Furthermore, there is abundant literature on potential long-term complications of these devices, including respiratory depression, catheter migration, and intrathecal granuloma for ITP (4) and electrode migration for SCS (5). Post-operative infections are also a known concern for all implantable devices. There is less information, however, on the short-term, perioperative complications of ITP, SCS, and PNS implantation. The demographics and indications of the patient population undergoing these procedures are also not entirely clear. For example, common indications for SCS include failed back surgery syndrome, radicular pain, neuropathic pain, and peripheral vascular disease, including Raynaud's phenomena, non-operative limb ischemia, chronic angina, and Berger's Disease. Off label applications include visceral pain, peripheral neuralgia, and peripheral field nerve stimulation. This lack of clarity may be due to the myriad of specialists performing these operations, current limitations in data collection, multiple manufactures producing these devices, as well as pain and non-pain diagnoses prompting implantation.

The National Anesthesia Clinical Outcomes Registry (NACOR) of the Anesthesia Quality Institute (AQI) is a database with detailed information about anesthetics administered for a variety of procedures and includes data on complications. This registry has used its extensive and growing data to report on uncommon events, such as intra-operative cardiac arrest, across a range of operations (6).

This study is a retrospective, observational analysis of patient demographics and perioperative issues relating to ITP, SCS, and PNS implantation, performed both for pain management as well as for other conditions.

\section{Methods}

NACOR directly imports electronic data from participating anesthesia professional billing systems, anesthesia information management systems, anesthesia quality measurement systems, and hospital electronic record systems. The extent of the data for each case varies by institution. In some instances, a case may include only a required minimum of 20 variables; in other instances, a case may include information on every vital sign and medication administered (7).

For our analysis, we identified ITP, SCS, and PNS by current procedural terminology (CPT) code. For ITP we used the following codes: 52361, 62350, 62355, 62360, 62362 , and 62365. For SCS we used 63650, 63661, 63663, 63685 , and 63688-59. For PNS we used 64553, 64555, $64575,64581,65685,64590$, and 64595.

We queried variables relating to patient demographics, anesthetic type, anesthetic complications, procedure duration, and procedure location. Patient demographics included age, ASA physical status class, and gender. Mean age was calculated and standard deviation was provided. Age groups were stratified to: under one year old, 1 - 18 years old, 19 - 49 years old, 50 - 64 years old, 65 - 79 years old, and greater or equal to 80 years of age. ASA class VI was removed from the analysis. The mean of case duration in minutes was calculated and standard deviation was provided. Case duration was additionally stratified to: under 60 minutes, 60 - 120 minutes, and greater than 120 minutes. Primary anesthesia type included general, neuraxial, monitored anesthetic care, and sedation. Data related to the facility at which the procedure occurred was collected. This included university hospitals, large community hospitals (defined as having over 500 beds), medium community hospitals (100 - 500 beds), small community hospitals (< 100 beds), specialty hospitals, attached surgery centers, and freestanding surgery centers. To obtain the diagnoses associated with ITP, SCS, and PNS, the present investigation queried the most commonly reported ICD-9 diagnoses codes associated with these cases. Clinical outcomes occurring with each case were collected. The numerator was defined as the absolute count of the occurrence. The calculated denominator included all cases eligible to report that outcome in the NACOR. Percentages were calculated based on these values. For each surgical case type, only the 5 most commonly reported outcomes were reported. 


\section{REsults}

Our search yielded 12,611 ITP, 19,276 SCS, and 15,184 PNS cases from 2010 to 2014 . Overall, the number of these cases performed has increased over time, as depicted in Fig. 1a; the number of cases performed only for pain diagnoses is depicted in Fig. $1 \mathrm{~b}$.

The demographics of the patients undergoing these procedures are listed in Table 1. Gender was roughly evenly split for ITP and SCS cases, but for PNS cases, $81 \%$ of patients were female. The majority of patients across the 3 categories were ASA physical class 2 or 3, but ITP cases had the highest proportion of ASA physical class 3 patients.

The duration of ITP, SCS, and PNS procedures varied widely within each category, but ITP procedures were longer, with a mean case duration of 112 minutes. Across all categories, these operations were performed most often at medium-sized community hospitals of $100-500$ beds. University hospitals represented only from $6.4-15.6 \%$ of cases in this sample.

The anesthetic type for ITP, SCS, and PNS procedures is illustrated in Fig. 2. The most common anesthetics

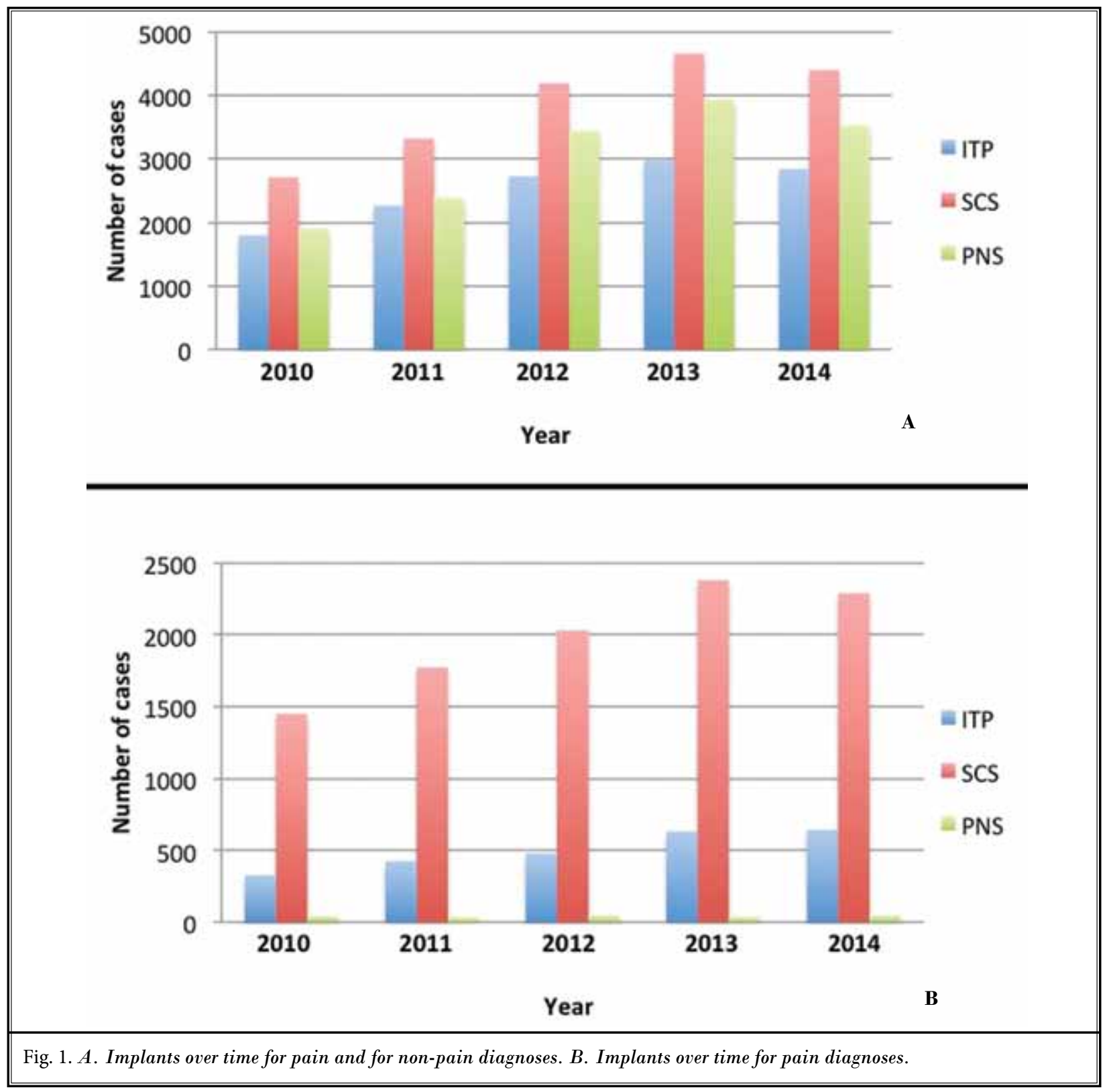


Table 1. Patient demographics and procedure information.

\begin{tabular}{|c|c|c|c|}
\hline & ITP & SCS & PNS \\
\hline Total number & 12611 & 19276 & 15184 \\
\hline Mean age (years) & $48.1+/-19.8$ & $55.1+/-16.3$ & $\begin{array}{c}58.0+1- \\
18.2\end{array}$ \\
\hline Gender: Male & $48 \%$ & $40 \%$ & $16 \%$ \\
\hline Gender: Female & $50 \%$ & $57 \%$ & $81 \%$ \\
\hline Not available & $2 \%$ & $3 \%$ & $3 \%$ \\
\hline ASA physical class 1 & $17 \%$ & $22.9 \%$ & $18.4 \%$ \\
\hline ASA physical class 2 & $21 \%$ & $33.4 \%$ & $33.4 \%$ \\
\hline ASA physical class 3 & $47 \%$ & $33.2 \%$ & $33.3 \%$ \\
\hline ASA physical class 4 & $5 \%$ & $1.4 \%$ & $1.5 \%$ \\
\hline Not available & $10 \%$ & $9.0 \%$ & $13.4 \%$ \\
\hline $\begin{array}{l}\text { Mean case duration } \\
\text { (minutes) }\end{array}$ & $112+/-92$ & $98+/-84.5$ & $88+/-99$ \\
\hline $\begin{array}{l}\text { University Hospital } \\
\text { Large Community }\end{array}$ & $14.9 \%$ & $6.4 \%$ & $15.6 \%$ \\
\hline $\begin{array}{l}\text { Hospital (over } 500 \\
\text { beds) }\end{array}$ & $21.9 \%$ & $10.1 \%$ & $13.5 \%$ \\
\hline Medium Community & & & \\
\hline $\begin{array}{l}\text { Hospital (100-500 } \\
\text { beds) }\end{array}$ & $35.8 \%$ & $33.6 \%$ & $32.9 \%$ \\
\hline Small Community & & & \\
\hline $\begin{array}{l}\text { Hospital (less than } \\
100 \text { beds) }\end{array}$ & $2.8 \%$ & $6 \%$ & $3.8 \%$ \\
\hline Specialty Hospital & $3.1 \%$ & $1.8 \%$ & $0.7 \%$ \\
\hline $\begin{array}{l}\text { Attached Surgery } \\
\text { Center }\end{array}$ & $3.2 \%$ & $7.3 \%$ & $5 \%$ \\
\hline $\begin{array}{l}\text { Freestanding } \\
\text { Surgery Center }\end{array}$ & $2.9 \%$ & $10.7 \%$ & $5.7 \%$ \\
\hline Not available & $15.4 \%$ & $24.1 \%$ & $23 \%$ \\
\hline
\end{tabular}

administered were general anesthesia and monitored anesthesia care; intravenous conscious sedation and neuraxial anesthesia represented only a small minority of cases. In this sample, general anesthetics were approximately twice as common for ITP procedures when compared for SCS and PNS procedures $(60.6 \%$ vs. $31.8 \%$ and $32.2 \%$, respectively). Conversely, monitored anesthesia care was less common for ITP procedures than for SCS and PNS procedures (13\% vs. $37.3 \%$ and $40.6 \%$, respectively).

The 5 most common perioperative outcomes for these procedures are detailed in Table 2. The same outcomes appeared for all the procedures, but were overall more common for ITPs, for which hemodynamic instability had an incidence of $13.9 \%$, extended Post Anesthesia Care Unit (PACU) stay was $4.5 \%$, nausea and vomiting was $2.3 \%$, and inadequate pain control was $2.2 \%$.

The diagnoses most frequently associated with ITP, SCS, and PNS are shown in Fig. 3A-C. For ITPs, the most common diagnosis associated with a procedure was an implant complication ( $n=2570)$, such as a mechanical complication related to the device or an infection or inflammatory reaction to the device. The next most common indication was spasticity $(n=1812)$, followed by back pain ( $n=1334$ ) and "other chronic pain" ( $n=$ 1292). ICD-9 codes for malignant pain ( $n=199)$ were associated with only a minority of ITP cases.

For SCS, the most common diagnosis was back pain without specific mention of radiculopathy $(n=5515)$,

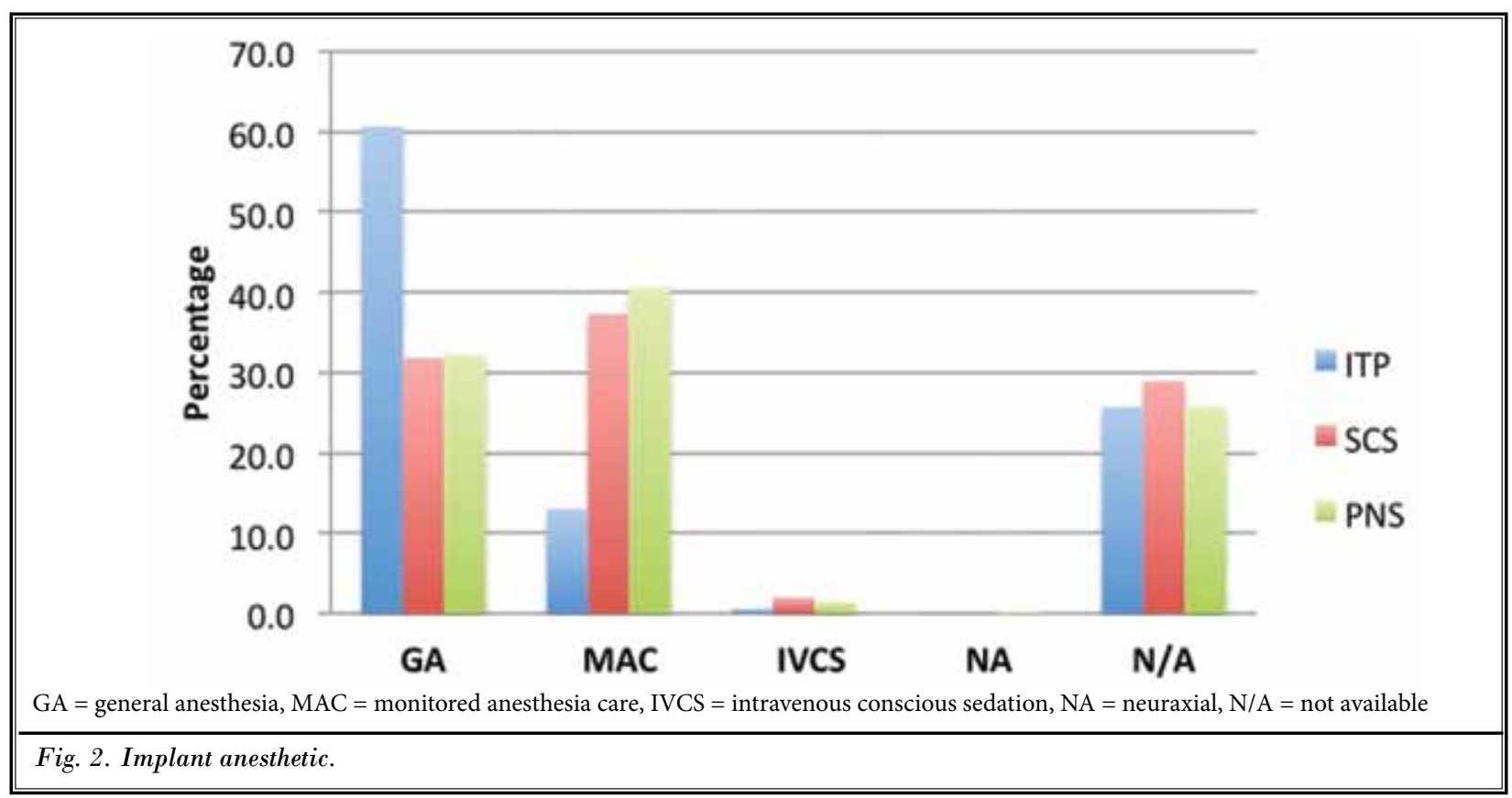


Table 2. Top outcomes by procedure.

\begin{tabular}{|c|c|c|c|c|c|}
\hline ITP & & SCS & & PNS & \\
\hline $\begin{array}{c}\text { Hemodynamic } \\
\text { instability }\end{array}$ & $13.9 \%$ & Case delayed & $9.8 \%$ & $\begin{array}{c}\text { Hemodynamic } \\
\text { instability }\end{array}$ & $8.4 \%$ \\
\hline Case delayed & $5.6 \%$ & $\begin{array}{c}\text { Hemodynamic } \\
\text { instability }\end{array}$ & $4.5 \%$ & Case delayed & $8.1 \%$ \\
\hline $\begin{array}{c}\text { Extended PACU } \\
\text { stay }\end{array}$ & $4.5 \%$ & Nausea/vomiting & $2.8 \%$ & $\begin{array}{c}\text { Inadequate pain } \\
\text { control }\end{array}$ & $3.6 \%$ \\
\hline Nausea/vomiting & $2.3 \%$ & $\begin{array}{c}\text { Inadequate pain } \\
\text { control }\end{array}$ & $2.2 \%$ & Nausea/vomiting & $2.4 \%$ \\
\hline $\begin{array}{l}\text { Inadequate pain } \\
\text { control }\end{array}$ & $2.2 \%$ & $\begin{array}{c}\text { Extended PACU } \\
\text { stay }\end{array}$ & $1.6 \%$ & $\begin{array}{c}\text { Extended PACU } \\
\text { stay }\end{array}$ & $0.7 \%$ \\
\hline
\end{tabular}

followed by radiculopathy or neuritis ( $n=2398)$. Implant complications $(n=1781)$ were the third most common diagnosis category, followed by complex regional pain syndrome (CRPS, $n=1068$ ).

For PNS, by far the most common diagnosis related to urinary dysfunction $(n=8745)$. Much less common were an implant complication $(n=1465)$ and defecation dysfunction $(n=1102)$. An even smaller group was for cystitis or painful bladder syndrome $(n=133)$.

\section{Discussion}

Benefits of using NACOR data to study ITP, SCS, and PNS procedures include the robust sample size, 47,071 cases in this study, and that data are automatically transmitted from participating institutions, minimizing bias that could result from selective sharing. In addition, NACOR applies standard anesthesia terminology upon transfer of the data from participating institutions to the registry. This data may also be used someday to estimate the denominator in closed claims analyses. This database is not, however, without shortcomings. First, not all institutions collect the same kind of data. One institution may, for instance, collect and therefore transmit data on the ICD-9 diagnosis codes associated with a particular procedure, while the other may not. Some institutions do not report clinical outcomes for every case to NACOR, making the sample size of assessing complications smaller and potentially more biased.

\section{Demographics and Procedure Data}

The high number and overall increase over time of ITP, SCS, and PNS procedures captured in the 5-year period of this study highlight the growing prominence of these interventions, whether for pain management or for other conditions. The majority of patients classified as ASA physical class 2 or 3-those with mild or severe systemic disease, respectively-in turn highlights that many of these patients, either due to their primary disease process or co-morbid conditions, are not ideal surgical candidates. In particular, patients undergoing ITP procedures were the most likely to have severe systemic disease. The severity of underlying disease in ITP patients may explain in part why ITP procedures were associated with more adverse outcomes overall than were SCS or PNS patients. The longer average procedure duration for ITPs (112 minutes) compared to SCS (98 minutes) and PNS (88 minutes) could reflect the larger pocket required for pumps. Despite the shorter time required for stimulators, these procedures are probably more technically difficult given the often time-consuming and challenging process of achieving proper stimulation.

The procedure data also indicated that the majority of ITP, SCS, and PNS procedures are performed not at academic medical centers but instead at community hospitals, in particular those with over 100 beds. One possibility for this trend is that academic medical centers are underrepresented in this sample. Surveys in the last 10 years, however, have shown that while approximately $5-10 \%$ of hospitals in the United States use electronic anesthesia record keeping (8), over $40 \%$ of academic medical centers in the United States use electronic anesthesia record keeping (9). 
A. ITP diagnoses based on ICD-9 code

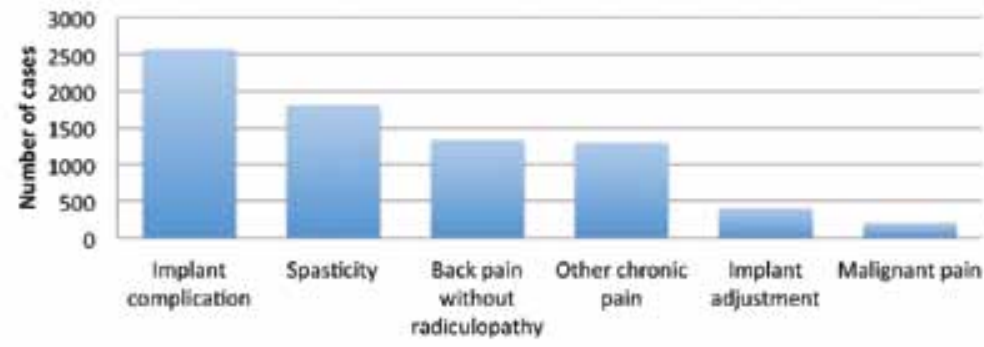

ICD-9 note: implant complication $=996.2,996.59,996.63$, spasticity $=343.9,728.85,7781.0$, backpain without radiculopathy $=722.8,722.83,724.5$, other chronic pain $=338.29,338.4$, implant adjustment $=$ V5309

\section{B. SCS diagnosis based on ICD-9 code}

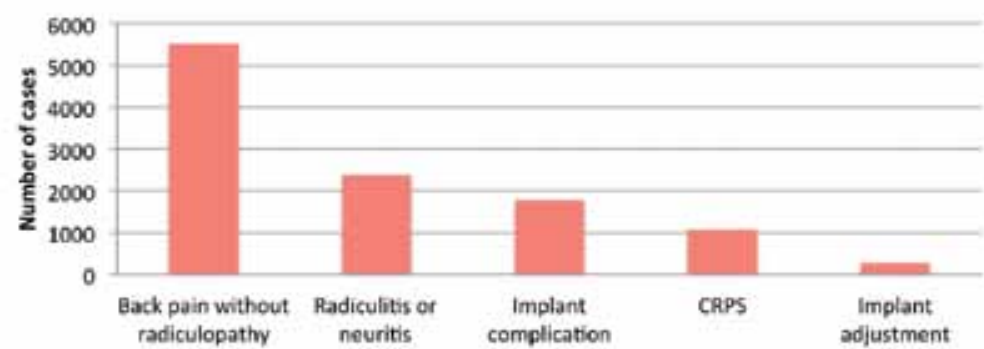

ICD-9 note: back pain without radiculopathy $=722.8,722.83,724.2,724.5$, radiculitis or neuritis $=355.9,723.1,723.4,724.4,729.3$, implant complication $=996.2,996.63,996.75$, CRPS $=337.20-22$, implant adjustment $=$ V5309

C. PNS diagnosis based on ICD-9 code

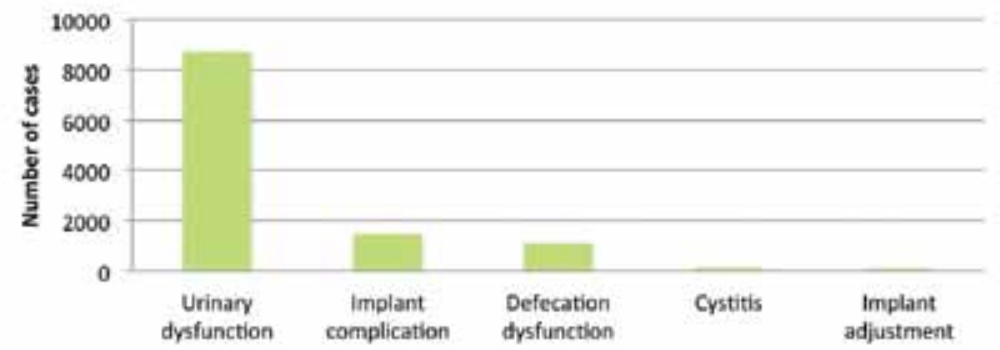

ICD-9 note: urinary dysfunction $=596.51,596.54,625.6,788.2,788.21,788.3,788.31,788.41$, $788.33,788.63$, implant complication $=996.2,996.3,996.39,996.63,996.75,996.76$, cystitis $=$ 595.1, implant adjustment $=$ V5309

Fig. 3. A. ITP diagnoses. B. SCS diagnoses. C. PNS diagnoses

\section{Implant Anesthetic Data}

For stimulator procedures, Monitored Anesthesia Care (MAC) was likely the more common anesthetic related to the benefit of intra-operative feedback from the patient to assure coverage of painful areas. For ITP procedures, general anesthesia was more likely than monitored anesthesia care. Cited benefits of MAC are the ability to receive intra-operative feedback from the patient during catheter placement, as well as reduction of post-operative cardiopulmonary complications. The relatively higher complications (e.g., hemodynamic instability) noted in this study for ITP procedures could, therefore, be related to preferred use of general anesthetics. There are, however, limitations in this dataset to making such conclusions. Additional information on the kind of procedure performed (e.g., a new ITP implant vs. a simple revision) as well as the specialty of the physician performing the procedure would be useful. There are also a significant proportion of procedures in this sample for which the anesthetic type is not known.

\section{Perioperative Outcome Data}

The most common adverse perioperative outcome for ITP, SCS, or PNS procedures was hemodynamic instability for ITP $(13.9 \%)$, which, as mentioned earlier, could be related to the severe systemic comorbidities in these patients or the preferred use of general anesthetics. Inadequate pain control across all procedures $(2.2-3.6 \%)$ highlights the known challenges of treating acute or chronic pain in post- 
surgical patients. This inadequacy of pain control could be a contributing factor for the extended PACU stays required for some of these patients (e.g., $4.5 \%$ of patients undergoing ITP procedures). It also highlights the possible challenges of performing such procedures at freestanding surgical centers or high-turnover venues.

\section{Associated Diagnosis Data}

The observation in the present investigation that implant complications were by far the most common diagnosis associated with ITP procedures might reflect the significant comorbidities in this patient population. Regardless of the cause, this data highlights the importance of discussing with patients the high likelihood of needing to return to the operating room for revisions. Such a conversation may be especially important in patients with malignant pain deciding whether to undergo implantation.

This diagnosis data is in line with the notion that ITP are commonly used to treat spasticity and SCS employed to treat post-laminectomy pain syndrome and CRPS. The relatively few cases of ITP associated with malignant pain could reflect a growing use of intrathecal drug delivery systems to treat non-malignant pain. In contrast, the paucity of ITP implanted for malignant pain could also reflect that such procedures are often done at academic medical centers, a practice setting representing only a minority of cases in this sample. As for stimulators, the strong association of urinary dysfunction with PNS likely explains why $81 \%$ of this patient population is female. It likely also suggests that the majority of PNS implants are not performed for pain management.

\section{Limitations of Data}

It should also be noted that "similar" procedures using NACOR data are not always "equal" and can potentially influence outcomes. For example, in a systematic review of 22 published reports from 1990 to 2002, a summary of complications from SCS for failed back surgery syndrome and complex regional pain syndrome was presented in Pain in 2004 (10). The authors reported an incidence of $34 \%$ with the majority of cases being revisions.10 In the same year, another literature review on the efficacy and safety of spinal cord stimulators was published that reviewed 51 publications in 20 years including 2,972 patients (11). This study reported lead migration was the most common adverse event with an incidence of $13.2 \%$; followed by lead breakage at $9.1 \%$, infection at $3.4 \%$, hardware malfunction at $2.9 \%$, and unwanted stimulation at $2.4 \%$. A subsequent review in 2008 provided additional insight by focusing in detail on the medical history of patients undergoing spinal cord stimulation (12). This study found, for instance, that $100 \%$ of patients identified with hematoma complications were on some type of anticoagulation therapy. Therefore, the more detailed diagnostic and patient data which should come with expanding diagnostic codes and improved electronic medical record documentation will likely be extremely useful in more precisely understanding outcomes data in the coming years.

\section{Conclusion}

The NACOR database is a source of diverse information on ITP, SCS, and PNS for physicians performing these procedures. In this sample, ITP procedures, performed on the patients with the most severe cormobidities and often-requiring general anesthesia, were the most likely to be associated with hemodynamic instability, inadequate pain control, and extended PACU stays. Complications relating to the ITP are also the most common reason for an operation. Additional information is needed on the detailed procedure performed (e.g., an implant vs. a revision), the surgical technique used, and the specific device implanted. Such information will likely become more available as resources like NACOR expand and as electronic medical record systems and coding become more elaborate. Hopefully, such databases will increasingly provide insight into the optimal treatment for intrathecal pump and nerve stimulator patients, both in the shorter term perioperative setting and into the outpatient setting. 


\section{References}

1. Manchikanti L, Singh V, Pampati V, Smith HS, Hirsch JA. Analysis of growth of interventional techniques in managing chronic pain in the Medicare population: A 10-year evaluation from 19972006. Pain Physician 2009; 12:9-34.

2. Metzner J, Posner KL, Lam MS, Domino KB. Closed claims' analysis. Best Practice Q Research Clinical Anesthesiology 2011; 25:267-273.

3. Rathmell JP, Michna E, Fitzgibbon DR et al. Injury and liability associated with cervical procedures for chronic pain. Anesthesiology 2011; 114:918-926.

4. Deer et al. Polyanalgesic consensus conference 2012: Recommendations to reduce morbidity and mortality in intrathecal drug delivery in the treatment of chronic pain. Neuromodulation. 2012; 15:467-482.
5. Bendersky D, Yampolsky C. Is spinal cord stimulation safe? A review of its complications. World Neurosurg 2014; 82:1359-1368.

6. Nunnally ME, O'Connor MF, Kordylewski H, Westlake B, Dutton RP. The incidence and risk factors for perioperative cardiac arrest observed in the national anesthesia clinical outcomes registry. Anesth Analg 2015; 120:364-370.

7. Dutton RP, DuKatz A. Quality improvement using automated data sources: The Anesthesia Quality Institute. Anesthesiology Clin 2011; 29:439-454.

8. Epstein $\mathrm{RH}$, Vigoda MM, Feinstein DM. Anesthesia information management systems: A survey of current implementation policies and practices. Anesth Analg 2007; 105:405-411.
9. Egger Halbeis $\mathrm{CB}$,Epstein $\mathrm{RH}$, Macario A, Pearl RG, Grunwald Z. Adoption of anesthesia information management systems by academic departments in the United States. Anesth Analg 2008; 107:1323-1329.

10. Turner JA, Loeser JD, Deyo RA, Sanders SB. Spinal cord stimulation for patients with failed back surgery syndrome or complex regional pain syndrome: $A$ systematic review of effectiveness and complications. Pain 2004, 108:137-147.

11. Cameron T. Safety and efficacy of spinal cord stimulation for the treatment of chronic pain: a 20-year literature review. J Neurosurg 2004, 100:254-267.

12. Deer TR, Stewart CD. Complications of spinal cord stimulation: Identification, treatment, and prevention. Pain Medicine 2008, 9:S93-S101. 